\title{
Skin Eruption and Gastrointestinal Symptoms as Presentation of COVID-19
}

\author{
Sherman Chu, BS; Luisa F. Christensen, MD; Dennis Vidmar, MD
}

\section{PRACTICE POINTS}

- Patients with coronavirus disease 2019 (COVID-19) typically present with fever, dry cough, dyspnea, and fatigue, but cutaneous manifestations also have been reported.

- Awareness of atypical presentations of COVID-19, including uncommon cutaneous manifestations, may identify more cases and help slow the expansion of this pandemic.
Recently, several cutaneous manifestations of coronavirus disease 2019 (COVID-19) have been reported. However, there is a paucity of published images. Those that have been published so far tend to fall under distinct morphologic categories. We present a 52-year-old patient with an ill-defined skin eruption that preceded mild gastrointestinal (Gl) symptoms and without respiratory symptoms who tested positive for COVID-19. With this case report, we widen the spectrum of the cutaneous manifestations of COVID-19 infection. Consequently, we propose an expansion of the criteria for COVID-19 testing when skin findings are associated with relatively mild Gl symptoms.

Cutis. 2020;106:309-310.

In December 2019, severe acute respiratory syndrome coronavirus 2 (SARS-CoV-2) started an outbreak of respiratory illnesses in Wuhan, China. The respiratory disease was termed coronavirus disease 2019 (COVID-19) and rapidly spread worldwide, resulting in a pandemic classification on March 11, 2020. ${ }^{1}$ Recently, several cutaneous manifestations of COVID-19 have been reported. Skin manifestations have been reported to be similar to other common viral infections. ${ }^{2}$ However, there is a paucity of published clinical images of more atypical presentations.

\section{Case Report}

A 52-year-old black man presented via urgent store-andforward teledermatology consultation from his primary care provider with a self-described "vesicular," highly pruritic rash of both arms and legs of 1 week's duration without involvement of the trunk, axillae, groin, face, genitalia, or any mucous membranes. He noted nausea, loss of appetite, and nonbloody diarrhea 4 days later. He denied fever, chills, dry cough, shortness of breath, or dyspnea. He had a history of hypertension and type 2 diabetes mellitus. There were no changes in medications; no outdoor activities, gardening, or yard work; no exposure to plants or metals; and no use of new personal care products.

The digital images showed zones of flesh-colored to slightly erythematous, somewhat "juicy" papules with some coalescence into ill-defined plaques. There were scattered foci of scale and hemorrhagic crust that involved both palms, forearms (Figure, A), and legs (Figure, B). There were no intact vesicles, and a herald patch was not identified. Vital signs at the time of imaging were normal, with the exception of a low-grade fever (temperature, $37.3^{\circ} \mathrm{C}$ ). Basic laboratory testing showed only mild leukocytosis with mild neutropenia and mild aspartate aminotransaminase elevation. A skin biopsy was not performed. Pulmonary imaging and workup were not performed because of the lack of respiratory symptoms.

The teledermatology differential diagnosis included a drug eruption, autosensitization eruption, unusual contact dermatitis, viral exanthem, secondary syphilis, and papular pityriasis rosea with an unusual distribution. The absence of changes in the patient's medication regimen and the

From the Department of Dermatology, Case Western Reserve University, Cleveland, Ohio. Mr. Chu also is from the Western University of Health Sciences, College of Osteopathic Medicine of the Pacific, Northwest, Lebanon, Oregon. Drs. Christensen and Vidmar also are from the Department of Dermatology, Louis Stokes Cleveland Department of Veterans Affairs Medical Center.

The authors report no conflict of interest.

Correspondence: Sherman Chu, BS, Department of Dermatology, Case Western Reserve University, 2109 Adelbert Rd, Cleveland, OH 44106 (sxc1407@case.edu).

doi:10.12788/cutis.0129 

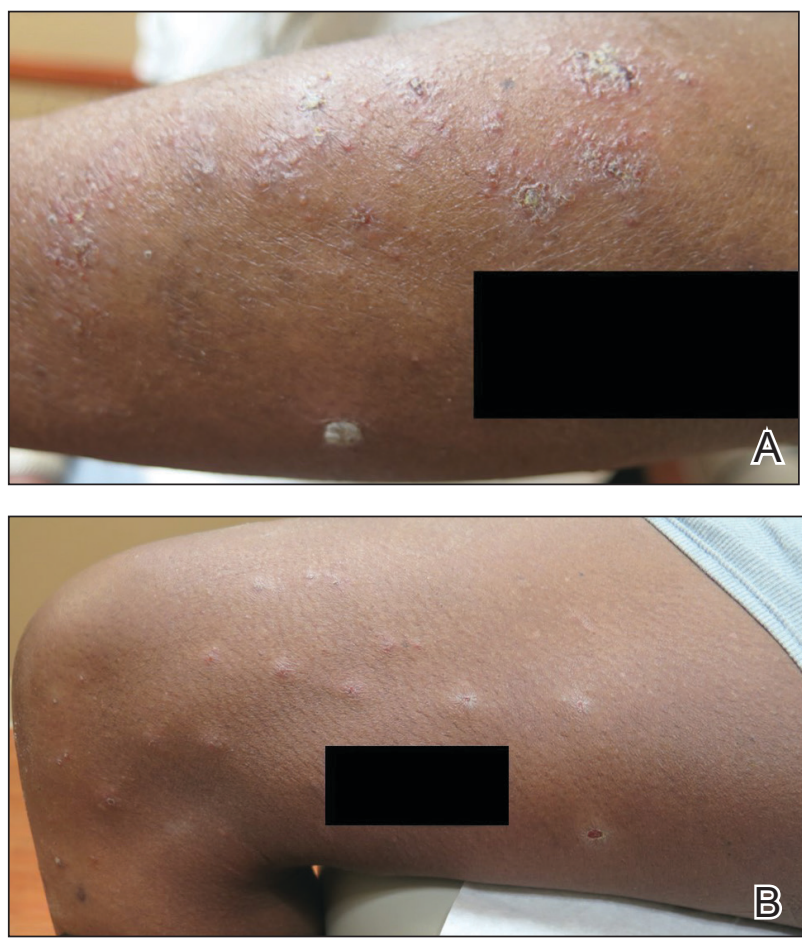

Coronavirus disease 2019. A, Slightly erythematous papules coalescing into ill-defined plaques with scale and hemorrhagic crust on the forearm. B, Flesh-colored papules with scale and erosion on the leg.

lack of outdoor activity in late winter made a drug eruption and contact dermatitis less likely, respectively. A rapid plasma reagin test drawn after disappearance of the rash was negative. Although the morphology of this eruption displayed some features of papular pityriasis rosea, this diagnosis was considered to be less likely given the presence of palmar involvement and the absence of any truncal lesions. This variant of pityriasis rosea is more commonly encountered in younger, darker-skinned patients.

Given the presence of an unusual rash on the extremities followed shortly by gastrointestinal (GI) symptoms and coupled with a low-grade fever, a nasopharyngeal swab was obtained to test for COVID-19 using a reverse transcriptase-polymerase chain reaction test. The results were positive.

The patient was treated with triamcinolone $0.1 \%$ slush (triamcinolone cream $0.1 \%$ mixed $1: 1$ with tap water) to the affected skin of the extremities 3 times daily, and he experienced a reduction in pruritus. He developed new lesions on the face and eyelids (not imaged) 2 days after teledermatology consultation. The facial involvement was treated with hydrocortisone cream 1\%. During the following week, the GI symptoms and skin eruption completely resolved. However, postinflammatory hyperpigmentation was observed in areas of the resolved papules and plaques. Over the course of this illness, the patient reported no respiratory symptoms.

\section{Comment}

Coronavirus disease 2019 is caused by SARS-CoV2, an enveloped, nonsegmented, positive-sense RNA virus of the coronavirus family. It is currently believed that SARS-CoV-2 uses the angiotensin-converting enzyme 2 receptor to gain entry into human cells, leading to infection primarily affecting the lower respiratory tract. ${ }^{3}$ Patients suspected of COVID-19 infection most often present with fever, dry cough, dyspnea, and fatigue, while GI symptoms such as nausea, vomiting, and diarrhea are uncommon. ${ }^{4}$ More recently, several reports describe a variety of skin findings associated with COVID-19. A current theory suggests that the virus does not directly target keratinocytes but triggers a systemic immune response, leading to a diversity of skin morphologies. ${ }^{5}$ The main types of described cutaneous findings include pseudochilblains, overtly vesicular, urticarial, maculopapular, and livedo/necrosis. ${ }^{6}$ Others have described petechial ${ }^{7}$ and papulosquamous eruptions. ${ }^{8}$ Most of these patients initially presented with typical COVID-19 symptoms and frequently represented more severe cases of the disease. Additionally, the vesicular and papulosquamous eruptions reportedly occurred on the trunk and not the limbs, as in our case.

This confirmed COVID-19-positive patient presented with an ill-defined vesicular and papulosquamous-type eruption on the arms and legs and later developed only mild GI symptoms. By sharing this case, we report yet another skin manifestation of COVID-19 and propose the possible expansion of testing for SARS-CoV-2 in patients presenting with rash and GI symptoms, which holds the potential to increase the identification of COVID-19 in the population, thereby increasing strict contact tracing and slowing the spread of this pandemic.

\section{REFERENCES}

1. Ng OT, Marimuthu K, Chia PY, et al. SARS-CoV-2 infection among travelers returning from Wuhan, China. $N$ Engl $\mathrm{J}$ Med. 2020;382:1476-1478.

2. Recalcati S. Cutaneous manifestations in COVID-19: a first perspective. J Eur Acad Dermatol Venereol. 2020;34:E212-E213.

3. Guo YR, Cao QD, Hong ZS, et al. The origin, transmission and clinical therapies on coronavirus disease 2019 (COVID-19) outbreakan update on the status. Mil Med Res. 2020;7:11.

4. Guan WJ, Ni ZY, Hu Y, et al. Clinical characteristics of coronavirus disease 2019 in China. N Engl J Med. 2020;382:1708-1720.

5. Gianotti R, Zerbi P, Dodiuk-Gad RP. Clinical and histopathological study of skin dermatoses in patients affected by COVID-19 infection in the Northern part of Italy. J Dermatol Sci. 2020;98:141-143.

6. Galván Casas C, Català A, Carretero Hernández G, et al. Classification of the cutaneous manifestations of COVID-19: a rapid prospective nationwide consensus study in Spain with 375 cases. Br J Dermatol. 2020;183:71-77.

7. Diaz-Guimaraens B, Dominguez-Santas M, Suarez-Valle A, et al. Petechial skin rash associated with severe acute respiratory syndrome coronavirus 2 infection. JAMA Dermatol. 2020;156:820-822.

8. Sanchez A, Sohier P, Benghanem S, et al. Digitate papulosquamous eruption associated with severe acute respiratory syndrome coronavirus 2 infection. JAMA Dermatol. 2020;156:819-820. 